\title{
Autosomal dominant cerebellar ataxia type III
}

INSERM

\section{Source}

INSERM. (1999). Orphanet: an online rare disease and orphan drug data base. Autosomal dominant cerebellar ataxia type III. ORPHA:94148

Autosomal dominant cerebellar ataxia (ACDA; see this term) type III is a group of neurodegenerative disorders characterized by mostly pure cerebellar syndromes with occasional non-cerebellar signs (e.g. pyramidal signs, peripheral neuropathy, writer's cramp) and includes spinocerebellar ataxia (SCA) type 5 (SCA5), SCA6, SCA11, SCA26, SCA30, and SCA31 (see these terms). 